\title{
LED-based photoacoustic imaging for early detection of joint inflammation in rodents: towards achieving 3Rs in rheumatoid arthritis research
}

Kalloor Joseph, Francis, Xavierselvan, Marvin, Kuniyil Ajith Singh, Mithun, Mallidi, Srivalleesha, van der Laken, Conny, et al.

Francis Kalloor Joseph, Marvin Xavierselvan, Mithun Kuniyil Ajith Singh, Srivalleesha Mallidi, Conny van der Laken, Fons van de Loo, Wiendelt Steenbergen, "LED-based photoacoustic imaging for early detection of joint inflammation in rodents: towards achieving $3 R s$ in rheumatoid arthritis research," Proc. SPIE 11240, Photons Plus Ultrasound: Imaging and Sensing 2020, 112400M (17 February 2020); doi: 10.1117/12.2545915

SPIE. Event: SPIE BiOS, 2020, San Francisco, California, United States 


\title{
LED-based photoacoustic imaging for early detection of joint inflammation in rodents - Towards achieving $3 R$ s in rheumatoid arthritis research
}

\author{
Kalloor Joseph Francis ${ }^{\mathrm{a}}$, Marvin Xavierselvan ${ }^{\mathrm{b}}$, Mithun Kuniyil Ajith Singh ${ }^{\mathrm{c}}$, Srivalleesha \\ Mallidi ${ }^{\mathrm{b}, \mathrm{d}}$, Conny van der Laken ${ }^{\mathrm{e}}$, Fons van de Loo ${ }^{\mathrm{f}}$, and Wiendelt Steenbergen ${ }^{\mathrm{a}}$ \\ ${ }^{a}$ Biomedical Photonic Imaging group, Technical Medical Center, University of Twente, The \\ Netherlands \\ bDepartment of Biomedical Engineering, Tufts University, Science and Technology Center, \\ Medford, USA \\ ${ }^{\mathrm{c}}$ Research and Business Development Division, Cyberdyne INC, Cambridge Innovation Center, \\ Rotterdam, The Netherlands \\ ${ }^{\mathrm{d}}$ Wellman Center for Photomedicine, Massachusetts General Hospital, Boston, USA \\ eDepartment of Rheumatology, Amsterdam Rheumatology and Immunology Center, \\ Amsterdam, The Netherlands \\ ${ }^{\mathrm{f}}$ Department of Rheumatology, Radboud University Medical Center, Nijmegen, The \\ Netherlands
}

\begin{abstract}
Synovial angiogenesis and hypoxia in the joints are biomarkers of Rheumatoid Arthritis (RA). The ability to probe blood and accurately estimate the oxygen concentration make multiwavelength Photoacoustic (PA) imaging a potential tool for early detection of RA. In this work, a multiwavelength LED-based PA imaging system was characterized based on its imaging depth, resolution and accuracy of oxygen saturation estimation. A multicenter 3R (Replace, Refine and Reduce) focused small animal study was conducted. The 3R strategy was devised by reusing RA animal models, in vivo imaging of healthy animals and ex vivo studies with human blood. RA animal cadaver models with different levels of synovial angiogenesis (control, positive RA and treated) were imaged and compared against results from a previous study using the same samples. An ex vivo PA oxygen saturation imaging using human blood was validated against oximeter readings and further verified it with in vivo animal studies. An imaging depth of $8 \mathrm{~mm}$ with an SNR of $10 \mathrm{~dB}$ was achieved for RA samples. A difference in PA intensity was observed for RA models compared to control and treated group. The PA oxygen saturation estimation correlates with oximeter readings, which is confirmed with in vivo studies. The results show the efficacy of the LED-based PA imaging system in RA diagnosis based on synovial angiogenesis and hypoxia. The imaging depth, resolution and oxygen saturation estimate are sufficient to differentiate RA samples from control. Our future work will focus on validating the method using arthritis animal models and demonstrating the 3R potential.
\end{abstract}

Keywords: Photoacoustic imaging, Ultrasound, Rheumatoid arthritis, Oxygen saturation, Small animal

\section{INTRODUCTION}

Inflammatory arthritis, such as rheumatoid arthritis (RA), is associated with a proliferation of synovial tissue and destruction of articular cartilage. Synovial angiogenesis is an important early symptom in the development and perpetuation of inflammatory arthritis. Angiogenesis from a combination of hypoxia and high metabolic demand increases the number of synovial vessels, which drives synovial infiltration and hyperplasia. The presence

Further author information:

K. J. Francis.: E-mail: f.kalloorjoseph@utwente.nl, Telephone: +31534891559

Photons Plus Ultrasound: Imaging and Sensing 2020, edited by Alexander A. Oraevsky, Lihong V. Wang, Proc. of SPIE Vol. 11240, 112400M - (C) 2020 SPIE · CCC code: 1605-7422/20/\$21 - doi: 10.1117/12.2545915 
of hyper vascularized synovial tissue is directly associated with disease activity. Ultrasound (US) imaging is one of the established imaging modalities which help with the diagnosis of arthritis by providing visualization of joint vascularity, synovitis, joint space narrowing, and joint erosions. US imaging offers dynamic assessment, high resolution for anatomical imaging, and high sensitivity in identifying the blood flow. It is also easily available and affordable, hence, widely accepted in the clinical evaluation of inflammatory arthritis. However, since it relies on measuring the speed of the blood flow relative to the probe, Doppler US is intrinsically more sensitive to the faster blood flow in relatively large vessels, while angiogenic microvessels with slow flow speeds, which are more relevant to inflammation, are often missed. Moreover, US imaging is not available for evaluating hypoxia, another important physiological biomarker of inflammatory arthritis.

Photoacoustic (PA) imaging is a promising biomedical imaging modality that has emerged over the last decade. ${ }^{1}$ This new imaging modality has shown promise in cancer imaging, brain functional imaging, guiding interventional procedures and monitoring of treatment and many more. ${ }^{2-5}$ One of the major advantages of PA is that physiological biomarkers of rheumatologic diseases such as neoangiogenesis, hypoxia, and hyperemia in the synovial tissues can be detected with multi-wavelength imaging. ${ }^{6}$ Therefore, PA imaging can be used for joint imaging to monitor rheumatologic conditions such as RA, osteoarthritis, seronegative spondyloarthropathies, crystal deposition diseases, and systemic lupus. ${ }^{7}$ Initial results have shown promise of using PA for RA imaging. ${ }^{8-11}$

In this work, we have used an LED-based hand-held PA imaging system for pre-clinical RA imaging on rodent models. We have developed a multicenter 3Rs (Replace, Refine and Reduce) strategy to refine and reduce animal use in RA-related research. We have studied the difference in synovial angiogenesis of small animal knee joint from three RA groups (negative, positive and treated) using PA imaging. The accuracy of oxygen saturation imaging has been tested with in vitro blood sample and further verified on a healthy mouse. This $3 \mathrm{R}$ strategy also enabled us to compare our results with that of the previous study using the same samples.

\section{MATERIALS AND METHODS}

We have focused on two key aspects of RA, namely synovial angiogenesis and hypoxia. Hence imaging blood vessels and obtaining accurate oxygen saturation using PA imaging were the major goals of this work. Additionally, we have checked whether the imaging depth is sufficient for the application. A handheld LED-based PA and US imaging system, AcousticX (Cyberdyne INC. Japan) was used for this work. ${ }^{12}$ We have used a 7 $\mathrm{MHz}$ (-6 dB bandwidth of 4-10 MHz) linear ultrasound transducer array with 128 elements for both US and PA imaging. LED units of $850 \mathrm{~nm}$ and a combined array of $750 / 850 \mathrm{~nm}$ were used for this study. A pulse width of $70 \mathrm{~ns}$ with pulse energy of $400 \mathrm{~mJ}$ and LED repetition rate of $4 \mathrm{kHz}$ were used for PA imaging. We have also used 64 frames averaging on-board within the DAQ and 6 frames during the reconstruction in the PC.

The multi-center 3Rs strategy was devised as follows. The hand-held PA and US imaging system from Cyberdyne was used at two academic research labs. At the University of Twente imaging facility, the phantom, in vitro blood oxygen saturation imaging and the ex vivo imaging of RA models were performed. RA animal models were provided by Radboud University Medical Center and Amsterdam Rheumatology and Immunology Center. These small animal RA models were used for different studies in the respective centers and sacrificed before they were used for PA imaging. The knee joints of the animals were dissected and fixated using formaldehyde. At the Tufts University, a healthy mouse was used for in vivo blood oxygen saturation imaging. In this way, no animals were sacrificed specifically for this study. In addition to that, all the centers shared data from individual studies to evaluate the performance of the PA imaging study.

First, the imaging probe was characterized for its resolution and penetration depth in phantom studies. A $30 \mu \mathrm{m}$ thick black suture wire (Vetsuture, France) was used as the PA target for characterization. Since hypoxia is another key biomarker for RA, we tested the capability of our proposed approach in imaging relative changes in oxygen saturation. Oxygen saturation imaging study was conducted on in vitro human blood. Human blood from a female volunteer was used for the oxygen saturation measurements with lithium heparin as an anticoagulant. Two nylon tubes of $0.5 \mathrm{~mm}$ inner diameter were imaged with one carrying normal blood with approximately $65 \%$ oxygen concentration and blood with varying levels of oxygen in the other tube. To change the oxygen saturation, the blood was first deoxygenated using sodium hydrosulfite. The blood was then exposed to air to 


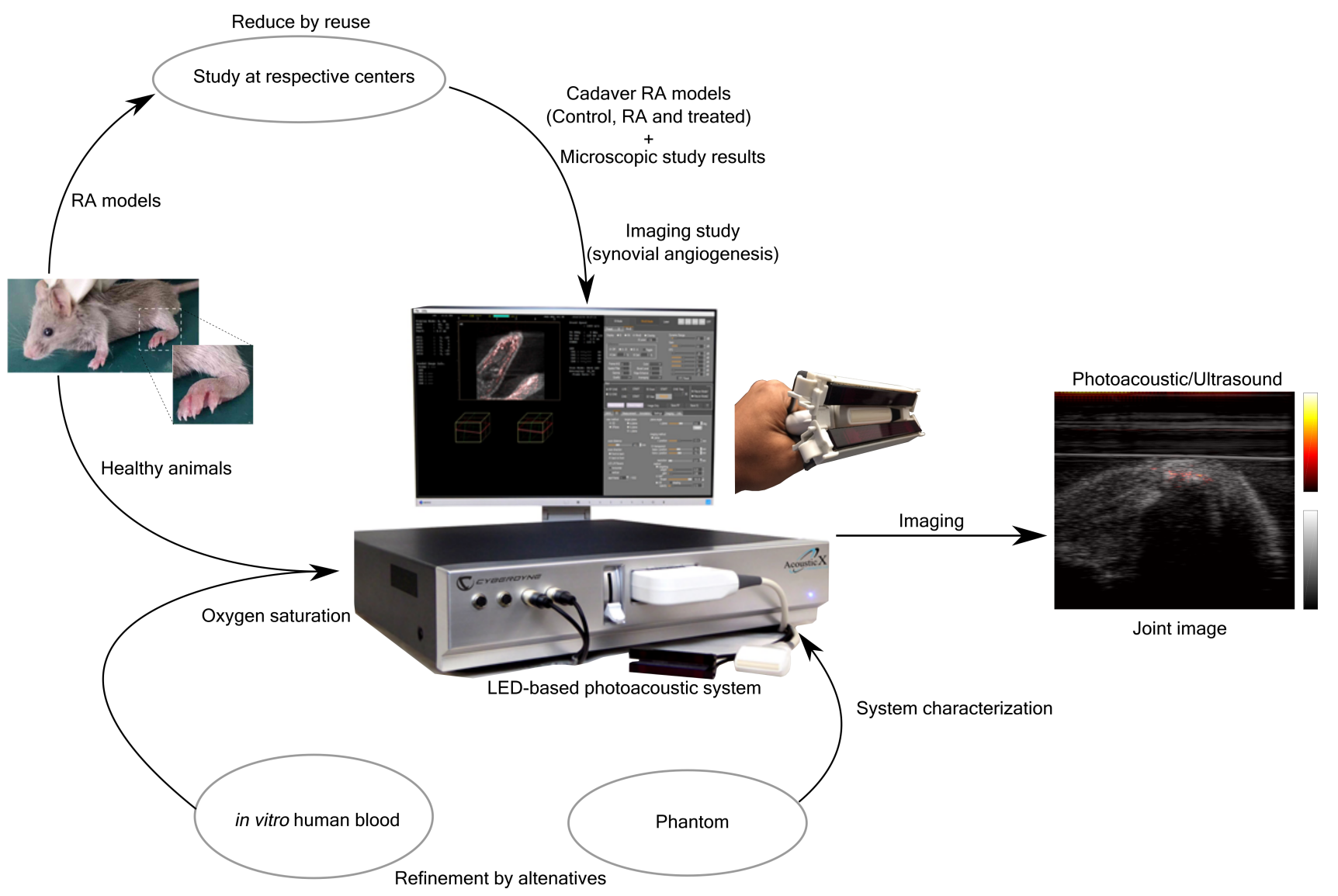

Figure 1. 3Rs strategy for preclinical photoacoustic/ultrasound RA imaging using portable LED-based system.

achieve different oxygen concentrations. The oxygen saturation in the blood was measured using an oxymeter, before and after the PA imaging. The two tubes carrying blood were imaged using the combination LED array $(750 / 850 \mathrm{~nm})$ and an oxygen saturation map was computed from the multi-wavelength PA images.

An in vivo oxygen saturation imaging was performed on the hind leg muscle of a mouse. The mouse was set to breath normal air and 100\% oxygen alternatively in a cyclic fashion. B-mode US and PA oxygen saturation imaging were collected in both oxygen concentration states. A larger blood vessel was selected as the region of interest and the average oxygen saturation in that region was computed.

RA was induced in mice using Freund's adjuvant with bovine collagen type II. The RA was confirmed with microscopic examination and a group was treated with anti-Interleukin-1. The animals were sacrificed after the study and the knee joints were fixated and transported to the imaging facility. The microscopic scores indicating redness, stiffness and the number of joints infected were also shared with the imaging group. At the imaging center, the knee joints were embedded in $3 \%$ agar phantom and the PA imaging was performed on the samples.

\section{RESULTS AND DISCUSSION}

The characterization of the probe provided a mean axial resolution of $240 \mu \mathrm{m}$ and a lateral resolution of $450 \mu \mathrm{m}$. In both phantom and small animal imaging, a minimum imaging depth of $8 \mathrm{~mm}$ was observed consistently. In the case of oxygen saturation, approximately $5 \mathrm{~mm}$ imaging depth was achieved. This imaging depth is considered sufficient for RA imaging of the finger joint.

Results from the blood oxygen saturation imaging are shown in Fig. 2. Fig. 2 a-c shows an oxygen saturation map of two tubes, one with normal blood (oxygen concentration approximately 65\%) and another with blood having 18\%, $43 \%$ and $87 \%$ oxygen concentration respectively. This correlates with the estimated 


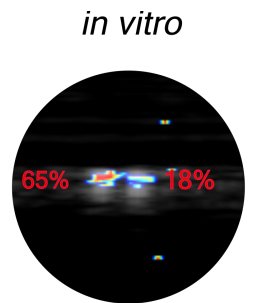

(a)

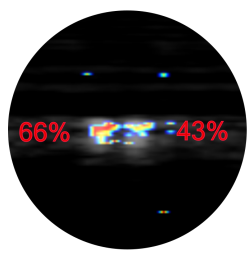

(b)

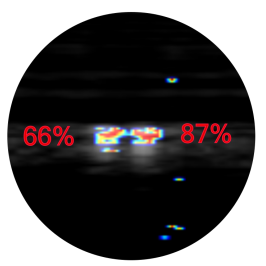

(c)

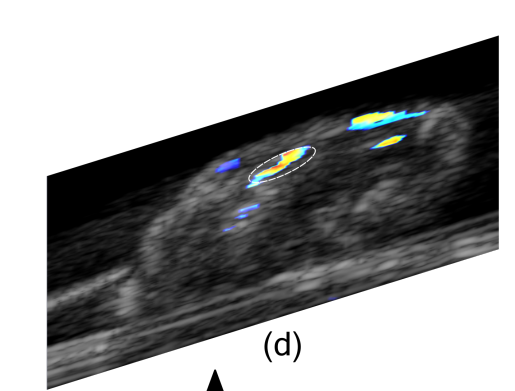

in vivo
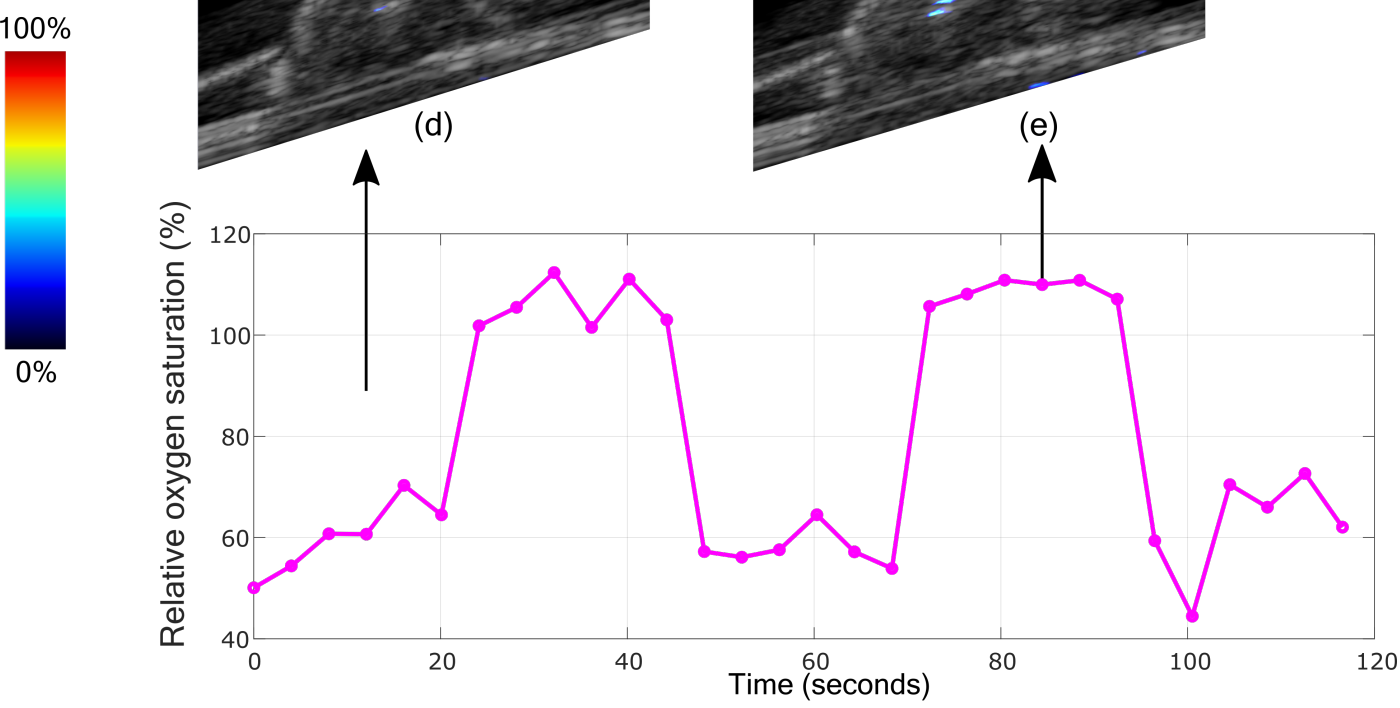

(f)

Figure 2. (a) - (c) In the in vitro study oxygen saturation map from three different oxygen concentrations in the tube on the right while keeping it unchanged in the left tube. (d) - (e) In vivo oxygen saturation images of the mice thigh muscle during high and low cycles of oxygen concentration. (f) Average oxygen saturation in the region of interested plotted over time.

oxygen saturation generated by the imaging system. Based on 28 measurements, the standard error of $8.4 \%$ was observed between the oximeter reading and oxygen saturation image formed by the system. Further, in vivo imaging on thigh muscle of the mouse is shown in Fig. 2 d-f. In the case of the mouse breathing normal air, an average oxygen concentration of $61.1 \%$ was observed and with $100 \%$ oxygen it was $105.3 \%$ using the system. This shows that the system can provide a fairly accurate estimate of oxygen saturation with in vivo imaging.

Figure. 3 shows PA and US imaging of mice knee. The images show joints from three RA types, negative (a - c), positive $(\mathrm{d}-\mathrm{f})$ and treated $(\mathrm{g}-\mathrm{i})$. PA images show the vascularization around the joint and the US images show the location of the joint. It can be observed that the number of blood vessels and their density is high in the case of diseased joint compared to negative and treated. The microscopic scores were assigned from 0 to 2 with 0 being RA negative and 2 being positive. The mean microscopic scores for negative knees, treated and positive were $0,0.3$ and 1.6 respectively. The PA images were normalized and the region of interest around the joint was selected. The mean PA value was calculated to be $0.47,0.42$ and 0.72 respectively for negative, treated and positive. The treated tissue was observed to have slightly lower vascularization seen in the PA image than the negative joint, while a significant difference was observed in the positive RA sample.

In this preliminary study, we have shown the potential of the handheld LED-based system in differentiating RA and negative joints. In the future, we will work with more number of samples to check the consistency of the proposed imaging method. Further, we intend to image the finger joints of human volunteers before considering any patient study. We are currently working towards a tomographic finger joint imager using the commercially available LED-based PA imaging system. ${ }^{13}$

\section{CONCLUSIONS}

The resolution, imaging depth and accuracy of oxygen saturation estimation were acceptable for monitoring rheumatoid arthritis in small animals. Through our results, we have shown that the early-stage rheumatoid 

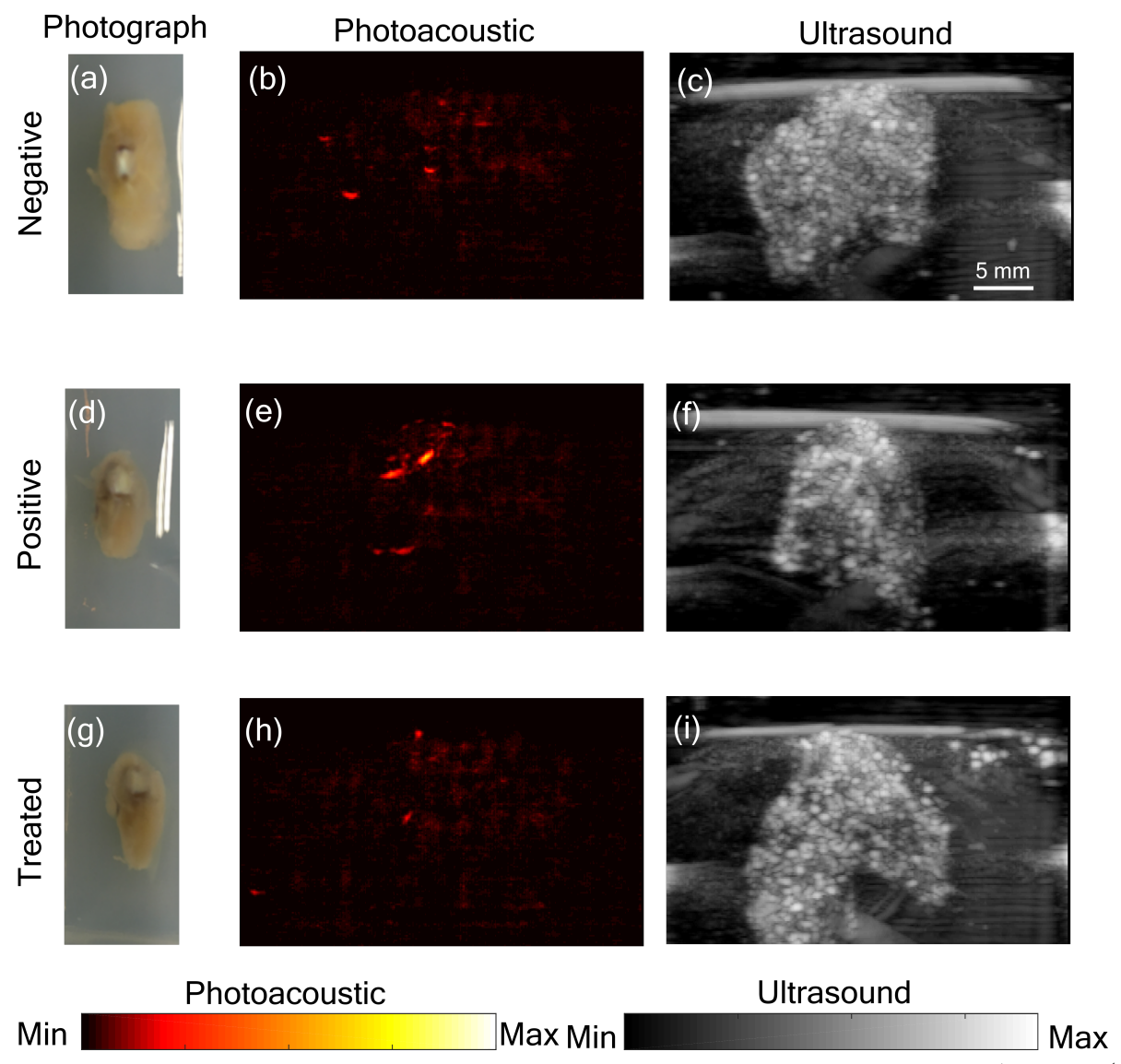

Figure 3. Photoacoustic and ultrasound maximum projection images of mouse knee of three RA states (negative, positive and treated).

arthritis changes such as synovial angiogenesis and hypoxia can be imaged using the LED-based photoacoustic imaging system. Our results give a direct confirmation that multispectral LED-based photoacoustics holds strong potential in early detection and staging of RA in animal studies, consequently achieving 3R's in RA-related pharmaceutical research.

\section{ACKNOWLEDGMENTS}

We acknowledge funding from NC3Rs as part of the CRACK-IT challenge, grant number CRACKITRT-P1-3 for supporting the project.

\section{REFERENCES}

[1] Yu, J., Nguyen, H. N. Y., Steenbergen, W., and Kim, K., "Recent development of technology and application of photoacoustic molecular imaging toward clinical translation," Journal of nuclear medicine 59(8), 12021207 (2018).

[2] Manohar, S. and Dantuma, M., "Current and future trends in photoacoustic breast imaging," Photoacoustics (2019).

[3] Francis, K. J., Chinni, B., Channappayya, S. S., Pachamuthu, R., Dogra, V. S., and Rao, N., "Characterization of lens based photoacoustic imaging system," Photoacoustics 8, 37-47 (2017).

[4] Wang, D., Wu, Y., and Xia, J., "Review on photoacoustic imaging of the brain using nanoprobes," Neurophotonics 3(1), 010901 (2016).

[5] Francis, K. J. and Manohar, S., "Photoacoustic imaging in percutaneous radiofrequency ablation: device guidance and ablation visualization," Physics in Medicine \& B Biology 64(18), 184001 (2019). 
[6] Rajian, J. R., Shao, X., Chamberland, D. L., and Wang, X., "Characterization and treatment monitoring of inflammatory arthritis by photoacoustic imaging: a study on adjuvant-induced arthritis rat model," Biomedical optics express 4(6), 900-908 (2013).

[7] Xu, G., Rajian, J. R., Girish, G., Kaplan, M. J., Fowlkes, J. B., Carson, P. L., and Wang, X., "Photoacoustic and ultrasound dual-modality imaging of human peripheral joints," Journal of biomedical optics 18(1), $010502(2012)$.

[8] Jo, J., Xu, G., Zhu, Y., Burton, M., Sarazin, J., Schiopu, E., Gandikota, G., and Wang, X., "Detecting joint inflammation by an led-based photoacoustic imaging system: a feasibility study," Journal of biomedical optics 23(11), 110501 (2018).

[9] van den Berg, P. J., Daoudi, K., Moens, H. J. B., and Steenbergen, W., "Feasibility of photoacoustic/ultrasound imaging of synovitis in finger joints using a point-of-care system," Photoacoustics 8, 8-14 (2017).

[10] van Es, P., Biswas, S. K., Moens, H. J. B., Steenbergen, W., and Manohar, S., "Initial results of finger imaging using photoacoustic computed tomography," Journal of biomedical optics 19(6), 060501 (2014).

[11] Nishiyama, M., Namita, T., Kondo, K., Yamakawa, M., and Shiina, T., "Ring-array photoacoustic tomography for imaging human finger vasculature," Journal of biomedical optics 24(9), 096005 (2019).

[12] Xia, W., Kuniyil Ajith Singh, M., Maneas, E., Sato, N., Shigeta, Y., Agano, T., Ourselin, S., J West, S., and E Desjardins, A., "Handheld real-time led-based photoacoustic and ultrasound imaging system for accurate visualization of clinical metal needles and superficial vasculature to guide minimally invasive procedures," Sensors 18(5), 1394 (2018).

[13] Francis, K. J., Boink, Y., Dantuma, M., Singh, M. K. A., Manohar, S., and Steenbergen, W., "Tomographic imaging with an led-based photoacoustic and ultrasound system," Biomed. Opt. Express (2020 - Accepted). 